\title{
A REVIEW: NUTRITIONAL BENEFITS OF PLANT-BASED SEED-NUT BUTTER
}

\author{
Harshita Joshi, Bhawna Bisht \\ Department of Food Technology, School of Applied and Life Sciences, Uttaranchal University, \\ Premnagar, Dehradun, Uttarakhand - 248007, India
}

\begin{abstract}
The popularity and development of dairy-free butter came into existence over past few years. The high fat content in dairy butter highlighted the need of various plant-based butters. They are rich in unsaturated fats that are said to lower the inflammation and risk of cardiovascular diseases. Seed butters like tahini, pumpkin seed butter, sunflower seed butter etc., have gained popularity due to its high protein and good fat content. Nut butters are also very popular because of their high content of essential fatty acids, fiber and protein. These plant-based butters are popular among the vegan culture. For this review paper, I worked with a combination of pumpkin seeds, sesame seeds and cashew nuts to make a highly nutritious plantbased butter that can be easily included in our diets.
\end{abstract}

Keywords: dairy-free, butter, vegan, pumpkin seed, sesame seed, cashew, nutritious

\section{INTRODUCTION}

Butter is a dairy product made up of fats, protein components of milk. Butter is most commonly used in form of spread on preferable breads. Butter is also widely used in baking and culinary arts. It consists of about $80 \%$ of fat that also gives it a very creamy texture. It can contribute to gaining weight as it contains very high calories in every teaspoon.

A plant-based butter is made from plant nuts like peanut butter, almond butter, cashew butter, hazelnut butter and seeds like pumpkin seeds, sesame seeds, sunflower seed butter etc. Although these seeds and nuts are also very high in fats like dairy butter, but they have higher percentage of unsaturated fats and contain good amounts of essential fatty acids and many minerals like zinc, calcium, magnesium etc. Thus, a dairy-free butter is a good replacement for dairy butter.

A seed butter is made up of seeds such as pumpkin seeds, chia seeds, sesame seed, flax seeds that is healthier option for human consumption. They are generally roasted and consumed. They are packed with high fibre and protein and a good proportion of fats, iron, calcium, magnesium, zinc etc. Sesame butter i.e., tahini is very famous among seed butters. Seed butter is a low-moisture semi- perishable product made of edible seeds, sweeteners, stabilizers and salt (sodium chloride). Roasting, grinding, chilling and tempering are the major processing steps of this product (MacDonald et al., 1985). Seed butter has grown to be a popular product in the market, being an alternative to margarine (Olga Radocaj, Dimic, and Vujasinovic, 2012).

Seed butter can be consumed by spreading on a toast, topping on crackers or for dipping vegetables. It also can be combined in bakery or confectionery products (Shakerardekani et al., 2013). Seed and nut butter as a combined food product will give a power-packed supply of nutritious elements like iron, manganese, zinc, magnesium, selenium, calcium and high amounts of protein, fibre and monounsaturated and polyunsaturated fats.

\section{IMPORTANCE OF PLANT-BASED BUTTER}

The mounting well-being concerns, with respect to the utilization of dairy butter because of its fat substance, have raised an alert to look for an elective plant-based margarines viz., nut butter and seed butter. So, we have made an attempt to combine the three separate seeds and nut i.e., pumpkin seed, sesame seed and cashew nut to make a true butter. Table 1 showing the nutritional facts of different plant-based butters.

The advantages of this spread over other all seed butters and all nut butters as follows:

- Benefits of many vitamins and minerals in one product

- Better supply for human nutrition

- No need of stabilizers and sweeteners

- Better sensory and physicochemical properties

\section{SOURCES AND IMPORTANCE OF PLANT-BASED BUTTER}

\subsection{Pumpkin seed}

Pumpkin (Cucurbita maxima) seeds are flat, enclosed in yellow-white husk commonly known as 'pepitas' (Amin and Thakur 2013; AbdelRahman 2006). Pumpkin seeds have long been valued as a mineral zinc source, and the World Health Organization recommends their use as a 
good way of obtaining this nutrient. In general, pumpkin seeds will be hostile to contagious and against microbial properties. They have lignans (pinoresinol, medioresinol, and lariciresinol) that display hostility to microbial and work exceptionally against viral properties. Pumpkin seed margarine has an extraordinary surface like smooth and a decent nutty flavor. As the dietary arrangement is high and exceptionally fibrous in nature, these spreads are utilized as discrete and nowdays being blended in with numerous other seeds like sesame or flax and almond margarines and so on to improve the flavour and upgrade the surface and dietary creation.

\subsection{Sesame seed}

Sesame (Sesamum indicum L.) from Pedaliaceae, is an important oil seed crop grown on tropical and the temperate zones of the world. (Biabani, and Pakniyat, 2008). Sesame is cultivated in several countries such as India, Sudan, China and Burma which are considered as the major producers (60\% of its total world production) (Abou-Gharbia et al., 2000). It is one of the oldest oil crops and is widely cultivated in Asia and Africa. (Ali et al., 2007). At least 4000 years ago, it was a highly prized oil crop of Babylon and Assyria. Sesame Seeds are best when used in unroasted form in tahini. Sesame seed paste is commonly called as tahini, which is a basic ingredient of hummus. It is used in dishes of vegan diets. It has a high source of calcium, phosphorus, manganese and potassium.

\subsection{Cashew}

The cashew tree (Anacardium occidentale) is a tropical evergreen tree that creates the cashew seed and cashew apple. It is additionally utilized in desserts, snacks, curries and so on. It is also prepared as cashew spread. Cashew is a significant nut among the tropics.

Cashew plays significant role as an edible nut and as a leading industrial product among tropical nuts (Chandrasekara and Shahidi 2011). Sweetened and flavoured spread from cashew kernel baby bits (CKBB) was prepared by (Nagaraja, 2003). It is extravagant in different supplements, including iron, copper, and phosphorus. The entirety of the fat in cashew spread is heart-solid unsaturated fat, which is incredible news for any individual who appreciates the smooth, rich taste as an option in contrast to nutty spread or customary margarine. Cashew margarine even contains less added substances.

Table 1: Nutritional Facts of Different Plant-Based Butters per 100g serving

Pumpkin Seed Butter Sesame Seed Butter Cashew Butter

\begin{tabular}{lccc}
\hline Calories (cal) & 571 & 595 & 167 \\
Fat (g) & 46.4 & 53.8 & 14 \\
Protein (g) & 35.7 & 17 & 5 \\
Carbohydrates (g) & 14.3 & 21.2 & 7.8 \\
Sugar (g) & 0 & 0.5 & $\sim$ \\
Fiber (g) & 7.1 & 9.3 & 0.6 \\
Cholesterol (mg) & 0 & 0 & 0 \\
Saturated Fats (g) & 10.7 & 7.5 & 2.8 \\
Trans Fatty Acids (g) & 0 & 0 & $\sim$ \\
Calcium (mg) & 71 & 426 & 12.2 \\
Iron, Fe (mg) & 12.9 & 9 & 1.4 \\
Potassium, K (mg) & $\sim$ & 414 & 155.1 \\
\end{tabular}


International Journal of Engineering Applied Sciences and Technology, 2020

Vol. 5, Issue 1, ISSN No. 2455-2143, Pages 471-473

Published Online May 2020 in IJEAST (http://www.ijeast.com)

\begin{tabular}{lccc}
\hline Magnesium (mg) & 429 & 95 & 73.3 \\
Phosphorus, P (mg) & 1071 & 0 & 129.8 \\
Zinc, Zn (mg) & 6.4 & 4.6 & 1.5 \\
Manganese (mg) & $\sim$ & 1.5 & 0.2 \\
Selenium, Se ( $\boldsymbol{\mu g})$ & $\sim$ & 34.4 & 3.3 \\
\hline
\end{tabular}

(Source: USDA)

\section{CONCLUSION}

Distinctive nut and seed margarines or spreads are set up by simmering the nut and seed, pounding, and blending in with sugar, salt, stabilizers and emulsifiers. Ideal simmering conditions are important for getting the nut and seed butter with better flavor. Level of pounding and procedure followed for granulating impacts the perfection and mouth feel of the butter.

\section{REFERENCE}

Abdel-Rahman, M. K. (2006). Effect of pumpkin seed (Cucurbita pepo L.) diets on benign prostatic hyperplasia (BPH): chemical and morphometric evaluation in rats. World J Chem, 1(1), 33-40.

Abou-Gharbia, H. A., Shehata, A. A. Y., \& Shahidi, F. (2000). Effect of processing on oxidative stability and lipid classes of sesame oil. Food research international, 33(5), 331-340.

Amin, T., \& Thakur, M. (2013). Research article cucurbita mixta (pumpkin) seeds-a general overview on their health benefits. Int J Recent Sci Res, 4(6), 846-854.

Chandrasekara, N., \& Shahidi, F. (2011). Antioxidative potential of cashew phenolics in food and biological model systems as affected by roasting. Food Chemistry, 129(4), 1388-1396.

Chien, Y. H. (2015). Shelf life extension of seed butter made with sesame, sunflower and pumpkin seeds (Doctoral dissertation, The Ohio State University).

Mangels, R. (2001). Guide to nuts and nut butters. Vegetarian J, 21, 20-23.

Naderikharaji, R., Pakniyat, H., \& Biabani, A. R. (2008). Effect of drought stress on photosynthetic rate of four rapeseed (Brassica napus) cultivars. Journal of Application Science, 8, 4460-4463.

Nagaraja, K. V. (2003). Preparation of spread from cashew kernel baby bits. Journal of food science and technology (Mysore), 40(3), 337-339.

Radocaj, O. F., Dimic, E. B., \& Vujasinovic, V. B. (2011). Optimization of the texture of fat-based spread containing hull-less pumpkin (Cucurbita pepo L.) seed press-cake. Acta Periodica Technologica, (42), 131-143.

Shakerardekani, A., Karim, R., Ghazali, H. M., \& Chin, N. L. (2013). Textural, rheological and sensory properties and oxidative stability of nut spreads - a review. International journal of molecular sciences, 14(2), 4223-4241. 